\title{
Potentialités Hypocholestérolémiantes D'euphorbia Heterophylla (L.) Klotz. \& Garcke (Euphorbiaceae) Chez Les Lapins Locaux (Oryctolagus Cuniculus L.)
}

\author{
Kouassi Gouha Firmin, (Doctorant, MSc)
}

Université Félix Houphouët-Boigny, 22 B.P. 582 Abidjan 22, Côte d'Ivoire Kouakou N'Goran David Vincent, (Maître-assistant, PhD, MSc, Ing. Agronome)

Institut National Polytechnique Félix Houphouët-Boigny, B.P. 1313

Yamoussoukro, Côte d'Ivoire

Coulibaly Seydou Ba Mohamed, (Doctorant, MSc)

Université Nangui Abrogoua, 02 B.P. 801 Abidjan 02, (Côte d'Ivoire

Koné Gningnini Alain, (Assistant, MSc, Ing. Agronome)

Institut National Polytechnique Félix Houphouët-Boigny, B.P. 1313

Yamoussoukro, Côte d'Ivoire)

Koffi Kouadio Frédéric, (Doctorant, $\mathrm{MSc}$ )

Kouassi Ahou Jeanne Félicité, (Doctorante, MSc)

Amoikon Kouakou Ernest, (Maître de Conférence)

Université Félix Houphouët-Boigny, 22 B.P. 582 Abidjan 22, Côte d'Ivoire

\section{Abstract}

Two tests to feed local rabbits (Oryctolagus cuniculus) were carried out to evaluate the hypocholesterolemic potentiality of Euphorbia heterophylla. The first test was carried out during 90 days (feeding (30 days), growing (30 days), and fattening (30 days)) on 45 rabbits. As for the second test, it was carried out during 30 days in the course of the fattening period on 27 rabbits. Animals were both subjected to continuous or alterned provisions during the last test of the two experimental diets. The first test or control (Pangran) was composed of Panicum maximum and granules "IVOGRAIN" for rabbit. The second test (Paneuph) was composed of Panicum maximum and Euphorbia heterophylla supplemented with granules "IVOGRAIN" for rabbit. In the second test, the experimental diets (Pangran and Paneuph) were isocaloric. The test 1 results, respectively, showed an important cholesterol-LDL reduction of $26 \%$ and $60 \%$ after 30 and 90 days of the Euphorbia heterophylla ingestion. However, when diets are isocaloric, the 
ingestion of this plant causes an important reduction of cholesterol-LDL of $59.2 \%$ and $65.4 \%$, after, respectively, 15 and 30 days of ingestion. From the results, deep studies should be carried out with the aim of evaluating the hypocholesterolemic effect from Euphorbia heterophylla which could contribute to prevent human cardiovascular diseases.

Keywords: Euphorbia heterophylla, ingestion, cholesterol-LDL, rabbit, hypocholesterolemic, cardiovascular diseases

\section{Résumé}

Deux essais d'alimentation des lapins locaux (Oryctolagus cuniculus) ont été conduits pour évaluer les potentialités hypocholestérolémiantes d'Euphorbia heterophylla. Le premier essai a été conduit durant 90 jours repartis en 30 jours d'allaitement, 30 jours de croissance et 30 jours d'engraissement, sur 45 lapins. Le second a été mené pendant 30 jours sur 27 lapins en phase d'engraissement. Les animaux ont été soumis à une distribution continue ou alternée de deux régimes expérimentaux. Le premier régime ou le témoin (Pangran), était composé de Panicum maximum et de granulé "IVOGRAIN" pour lapin. Le deuxième (Paneuph) était constitué de $P$. maximum et d'E. heterophylla supplémenté de granulé "IVOGRAIN" pour lapin. Dans le second essai, les régimes testés (Pangran et Paneuph) étaient iso-caloriques. Les résultats de l'essai indiquent une réduction significative du cholestérol LDL de $26 \%$ et $60 \%$ respectivement, après 30 jours et 90 jours d'ingestion d'E. heterophylla par le lapin. Cependant, lorsque les régimes sont iso-caloriques, l'ingestion de cette plante induit une réduction significative du cholestérol LDL de 59,2 \% et de $65,4 \%$ respectivement après 15 jours et 30 jours d'ingestion par le lapin. Au regard de ces résultats, des travaux approfondis, devraient être entrepris afin de mieux évaluer l'effet hypocholestérolémiant d'E. heterophylla, qui pourrait participer à la prévention des maladies cardiovasculaires chez l'Homme.

Mots-clé: Euphorbia heterophylla, ingestion, cholestérol-LDL, lapin, hypocholestérolémie, maladies cardiovasculaires

\section{Introduction}

Les maladies cardiovasculaires sont la première cause de mortalité dans le monde (FAO, 2015). Dans l'ensemble des pays en voie de développement, elles provoquent deux fois plus de décès que le VIH/SIDA, le paludisme et la tuberculose réunis (Abegunde et al., 2007). Une des causes majeures de ces maladies est l'augmentation des lipoprotéines (LDL), transporteurs plasmatiques du cholestérol du foie vers les organes "mauvais cholestérol" et/ou la réduction des lipoprotéines (HDL), transporteurs 
plasmatiques du cholestérol des organes vers le foie "bon cholestérol". Bien que le cholestérol soit indispensable à la constitution de la couche lipidique des membranes plasmiques, son taux élevé et sa mauvaise répartition dans l'organisme, induisent de graves effets sur la santé (Callias, 2007). Les stratégies de lutte contre l'hypercholestérolémie utilisent plusieurs substances hypocholestérolémiantes telles que les phytostérols (Dylan et Peter, 2010), les acides gras polyinsaturés (AGPI) oméga 3 (Barcelo-Coblijn et Murphy, 2009), les polyphénols (Faleye et al., 2012) et les statines (Scheen, 2011).

En Afrique, où $80 \%$ de la population a recours à la médecine traditionnelle (OMS, 2003), le traitement de l'hypercholestérolémie est basé essentiellement sur des substances d'origine végétale administrées sous forme de tisane des feuilles, d'écorces ou des racines (N'Guessan et al., 2011). En effet, les plantes ont l'avantage de contenir différentes substances hypocholestérolémiantes, entre autres, les phytostérols, les polyphénols et les AGPI oméga 3 (Iserin et al., 2001). Parmi ces plantes, figurerait Euphorbia hirta, une euphorbe bien connue en Afrique et en Asie pour ces bienfaits sur la santé (Huang et al., 2012). Récemment, il a été montré que Euphorbia heterophylla, une plante adventice tropicale, dont la teneur en oméga 3 (53\% des acides gras totaux), (Kouakou et al., 2013; 2015), induisait chez les lapins qui la consommait un enrichissement du muscle de la cuisse en AGPI oméga 3 (Ahongo et al., 2016; Kouakou et al., 2016). En effet, un régime supplémenté en $E$. heterophylla a, respectivement, fait doubler et quadrupler la teneur en acide alpha linolénique (C18:3 n-3) après 30 jours et 90 jours d'ingestion par rapport au régime contrôle. Par ailleurs, les ratios acide alpha linoléique sur acide alpha linolénique (C18:2 n-6/C18:3 n-3) étaient de 2,2 et de 5,1 respectivement après 90 et 30 jours de supplémentation (Ahongo et al., 2016). Aussi, afin d'évaluer l'impact d'E. heterophylla sur la cholestérolémie (cholestérol total, cholestérol-HDL et cholestérol-LDL) des lapins, deux essais ont été réalisés.

\section{Matériel et méthodes}

\section{Animaux et aliments expérimentaux}

Les lapins étaient de race hybride locale (Oryctolagus cuniculus). Ils provenaient de la ferme de production de l'Institut National Polytechnique Félix Houphouët-Boigny de Yamoussoukro (INP-HB). Le premier essai ou "Essai 1 » comportait 45 lapereaux âgés d'un jour et le second essai ou «Essai 2 », 27 jeunes lapins âgés de 66 jours. Leurs poids moyens étaient respectivement de $60 \pm 1,4 \mathrm{~g}$ et $1,09 \pm 0,1 \mathrm{~kg}$. Le matériel végétal était constitué de feuilles et de tiges de l'herbe de Guinée (Panicum maximum Jacq. var G23), et de l'herbe de lait (E. heterophylla L.). Ces fourrages ont été récoltés chaque matin dans le périmètre de l'INP-HB puis distribués aux animaux sous forme fraîche. En plus de ces aliments, des granulés pour lapin 
de type croissance ont été achetés dans le commerce (SIPRA - IVOGRAIN). Des échantillons des trois aliments expérimentaux ont été prélevés pour la détermination de leurs compositions chimiques (matière sèche analytique, protéine brute, fibre brute et cendre brute) telle que recommandé par l'Association Officielle des Chimistes Analytiques (AOAC, 2006).

\section{Conduite des essais}

Afin d'évaluer les potentialités hypocholestérolémiantes d'E. heterophylla sur les paramètres biologiques chez le lapin, trois traitements expérimentaux (MOD1, MOD2 et MOD3) ont été définis sur la base des distributions des deux régimes expérimentaux aux lapins (Pangran et Paneuph). Les fourrages étaient distribués ad libitum de telle sorte qu'il y ait au moins $15 \%$ de refus (Tableau 1). Dans l'essai 1, les traitements expérimentaux MOD1 et MOD2 ont consisté respectivement à la distribution des régimes Pangran et Paneuph durant l'allaitement (30 jours), la croissance (30 jours) et l'engraissement (30 jours). Quant au traitement expérimental MOD3, il a consisté en la distribution du régime expérimental Pangran durant l'allaitement et la croissance et la distribution du régime expérimental Paneuph en phase d'engraissement (Tableau 1). Dans 1'essai 2, la distribution des traitements MOD1 et MOD2 a été faite uniquement durant la période d'engraissement (30 jours). Quant au traitement expérimental MOD3, il a consisté en la distribution du régime expérimental Pangran durant les 15 permiers jours de la période d'engraissement et la distribution du régime expérimental Paneuph sur les 15 derniers jours. Les quantités d'aliments distribués ont été réajustées chaque trois jours, afin que les régimes expérimentaux soient iso-caloriques.

Tableau 1 : Quantités (g) d'aliments expérimentaux frais distribués durant les essais en fonction des régimes expérimentaux testés et des stades physiologiques

\begin{tabular}{|c|c|c|c|c|c|c|c|}
\hline \multirow[b]{3}{*}{ Essais } & & \multirow[b]{3}{*}{$\begin{array}{l}\text { Durée (j) } \\
\text { ingestion }\end{array}$} & \multicolumn{5}{|c|}{ Régimes alimentaires } \\
\hline & \multirow[b]{2}{*}{$\begin{array}{c}\text { Stades } \\
\text { physiologiques }\end{array}$} & & \multicolumn{3}{|c|}{ Paneuph } & \multicolumn{2}{|c|}{ Pangran } \\
\hline & & & $\begin{array}{l}\text { Panicum } \\
\text { maximum }\end{array}$ & $\begin{array}{c}\text { Euphorbia } \\
\text { heterophylla }\end{array}$ & $\begin{array}{c}\text { Granulés } \\
\text { pour } \\
\text { lapin }\end{array}$ & $\begin{array}{l}\text { Panicum } \\
\text { maximum }\end{array}$ & $\begin{array}{c}\text { Granulés } \\
\text { pour } \\
\text { lapin }\end{array}$ \\
\hline \multirow{3}{*}{1} & Allaitement & 1 à 30 & $\begin{array}{c}300 \text { à } \\
600\end{array}$ & 400 à 1200 & 50 & $\begin{array}{c}300 \text { à } \\
600\end{array}$ & $\begin{array}{c}200 \text { à } \\
300\end{array}$ \\
\hline & Croissance & 31 à 60 & 50 à 100 & 200 à 400 & 25 à 50 & 50 à 100 & 75 à 120 \\
\hline & Engraissement & 61 à 90 & 100 & 400 à 500 & 50 & 100 & $\begin{array}{c}120 \text { à } \\
150\end{array}$ \\
\hline \multirow[b]{2}{*}{2} & \multirow[b]{2}{*}{ Engraissement } & 1 à 15 & 100 & 400 à 500 & 50 & 100 & $\begin{array}{c}120 \text { à } \\
150\end{array}$ \\
\hline & & 16 à 30 & 100 & 400 à 500 & 50 & 100 & $\begin{array}{c}120 \text { à } \\
150\end{array}$ \\
\hline
\end{tabular}


En début de chaque période d'élevage, les animaux ont été soumis à un traitement anticoccidien (Narcox : $1 \mathrm{~g} /$ litre d'eau) à titre préventif pendant trois jours et un déparasitant (Pipérazine 100\%: 1g par $10 \mathrm{~kg}$ ). L'eau de boisson a été distribuée à volonté deux fois par jour ( 8 h et 17h 30). Les animaux ont reçu par cage chaque semaine une orange récoltée dans le périmètre de l'INP-HB comme apport en vitamine C. Les ingestions volontaires d'aliment ont été quantifiées. Les animaux ont été pesés au début de l'essai, puis toutes les semaines jusqu'à la pesée finale à jeun.

A la fin de chaque essai, les six plus lourds lapins issus de chaque traitement (MOD1, MOD2 et MOD3) ont été étourdis avant d'être saignés au niveau de la carotide. Le sang frais carotidien a été recueilli dans des tubes rouges secs. Après coagulation et centrifugation à 4000 tours par minute pendant dix minutes, le sérum frais obtenu a été transvasé dans des tubes secs pour le dosage du cholestérol total, cholestérol-HDL triglycérides à l'aide d'un spectrophotomètre UV visible HITACHI 704 selon les longueurs d'onde adaptées et selon les méthodes de calcul contenu dans le protocole d'utilisation indiqué par les kits de dosage de référence (MEDIFF, France) (Tableau 2). Le cholestérol LDL a été déterminé par calcul (Friedwald et al., 1972).

Tableau 2 : Méthodes d'analyse des métabolites

\begin{tabular}{llc}
\hline Paramètres & Méthodes & $\lambda(\mathrm{nm})$ \\
\hline Cholestérol (CT) & Colorimétrique enzymatique CHOD-PAP & 510 \\
Cholestérol HDL (C-HDL) & Précipitation PEG 6000 & 510 \\
Triglycérides (TG) & Colorimétrique enzymatique GPO-PAP & 510 \\
Cholestérol LDL (C-LDL) & C-LDL = CT - C-HDL - TG/5 & \\
\hline
\end{tabular}

\section{Analyses statistiques}

A la fin de l'étude, les valeurs moyennes par traitement des paramètres étudiés ont été déterminées. Elles ont été soumises à une analyse de variance à un facteur au seuil de signification de 5\% à l'aide du logiciel $\mathrm{R}$ 3.3.2.

\section{Résultats}

La composition chimique, le profil en acides gras des aliments expérimentaux et les estimations des énergies métabolisables sont présentés dans le tableau 3.

\section{Effet des régimes sur l'ingestion alimentaire}

Les valeurs moyennes de l'ingestion journalière de la matière sèche (IVJMS) des aliments et régimes sont présentées dans le tableau ${ }^{4}$. Les 
valeurs moyennes d'IVJMS des aliments différaient selon les régimes alimentaires et selon le stade physiologique étudiés $(\mathrm{P}<0,05)$. Les ingestions volontaires journalières de matière sèche d'E. heterophylla représentaient respectivement $52 \%$ et $46 \%$ des ingestions volontaires journalières de matière sèche des régimes expérimentaux au cours des essais 1 et 2 . Les profils d'acides gras des aliments étaient différents. En effet, les proportions en acide laurique (C12:0) et en acide myristique (C14:0) étaient plus élevées dans le granulé pour lapin. Par ailleurs, les proportions en acide palmitoléique (C16:1 n-7) et en acide oléique (C18:1 n-9) étaient plus élevées dans $P$. maximum. Quant à la proportion en acide gras $\alpha$-linolénique (C18:3 n-3) de E. heterophylla, elle était 28 fois plus élevée que dans le granulé pour lapin et 2 fois plus élevée que dans le $P$. maximum.

Tableau 3 : Composition chimique et profil en acides gras des différents aliments étudiés

\begin{tabular}{|c|c|c|c|}
\hline \multirow{2}{*}{ Compositions (\%MF) } & \multicolumn{3}{|l|}{ Aliments étudiés } \\
\hline & $\begin{array}{l}\text { Euphorbia } \\
\text { heterophylla }\end{array}$ & $\begin{array}{l}\text { Granulés pour } \\
\text { lapin }\end{array}$ & $\begin{array}{l}\text { Panicum } \\
\text { maximum }\end{array}$ \\
\hline Matière sèche (MS) & 18,4 & 89,9 & 25,7 \\
\hline Matière organique (MO) & 89,7 & 90,4 & 88,2 \\
\hline Matière minérale (MM) & 12,5 & 9,6 & 11,8 \\
\hline Matière grasse (MG) & 7,2 & 7,4 & 2,5 \\
\hline Cellulose brute (CB) & 21 & 14,2 & 18,8 \\
\hline $\begin{array}{ll}\begin{array}{l}\text { Energie } \\
\left(\mathrm{kcal} . k g^{-1} \mathrm{MS}\right)\end{array} & \text { métabolisable } \\
\end{array}$ & 1970 & 2702 & 1938 \\
\hline \multicolumn{4}{|l|}{ Acides gras } \\
\hline$\sum$ AGS & 22,6 & 40,6 & 25,4 \\
\hline $\mathrm{C} 12: 0$ & 0,1 & 14,5 & 0,1 \\
\hline C14:0 & 0,4 & 5,4 & 0,2 \\
\hline C16:0 & 19,6 & 18,8 & 22,5 \\
\hline C18:0 & 2,5 & 1,9 & 2,6 \\
\hline$\sum$ AGMI & 3,6 & 20,4 & 22,6 \\
\hline $\mathrm{C} 16: 1 \omega 7$ & 0,4 & 0 & 0,51 \\
\hline $\mathrm{C} 16: 1 \omega 9$ & 0,9 & 0,1 & 1,8 \\
\hline $\mathrm{C} 18: 1 \omega 7$ & 1 & 1,1 & 0,1 \\
\hline $\mathrm{C} 18: 1 \omega 9$ & 2,2 & 19,2 & 20,2 \\
\hline$\sum$ AGPI & 67,2 & 37,9 & 48,4 \\
\hline $\mathrm{C} 18: 2 \omega 6$ & 10,7 & 35,9 & 21,4 \\
\hline $\mathrm{C} 18: 3 \omega 3$ & 56,5 & 2 & 27 \\
\hline AGPI/AGS & 3 & 0,9 & 1,9 \\
\hline $\mathrm{C} 18: 2 \omega 6 / \mathrm{C} 18: 3 \omega 3$ & 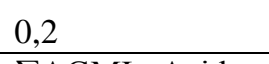 & 18,1 & 0,8 \\
\hline
\end{tabular}


$\omega 3$ moyen ; Matière sèche : matière sèche en $\%$ de la matière fraiche ; EM a été calculée à partir de la formule suivante : $\mathrm{EM}\left(\mathrm{kcal}_{\mathrm{kg}}{ }^{-1} \mathrm{MS}\right)=3951+54,4 \mathrm{MG}-88,7 \mathrm{CB}-40,8 \mathrm{MM}$

Tableau 4 : Ingestions volontaires journalières de la matière sèche des animaux au cours des essais

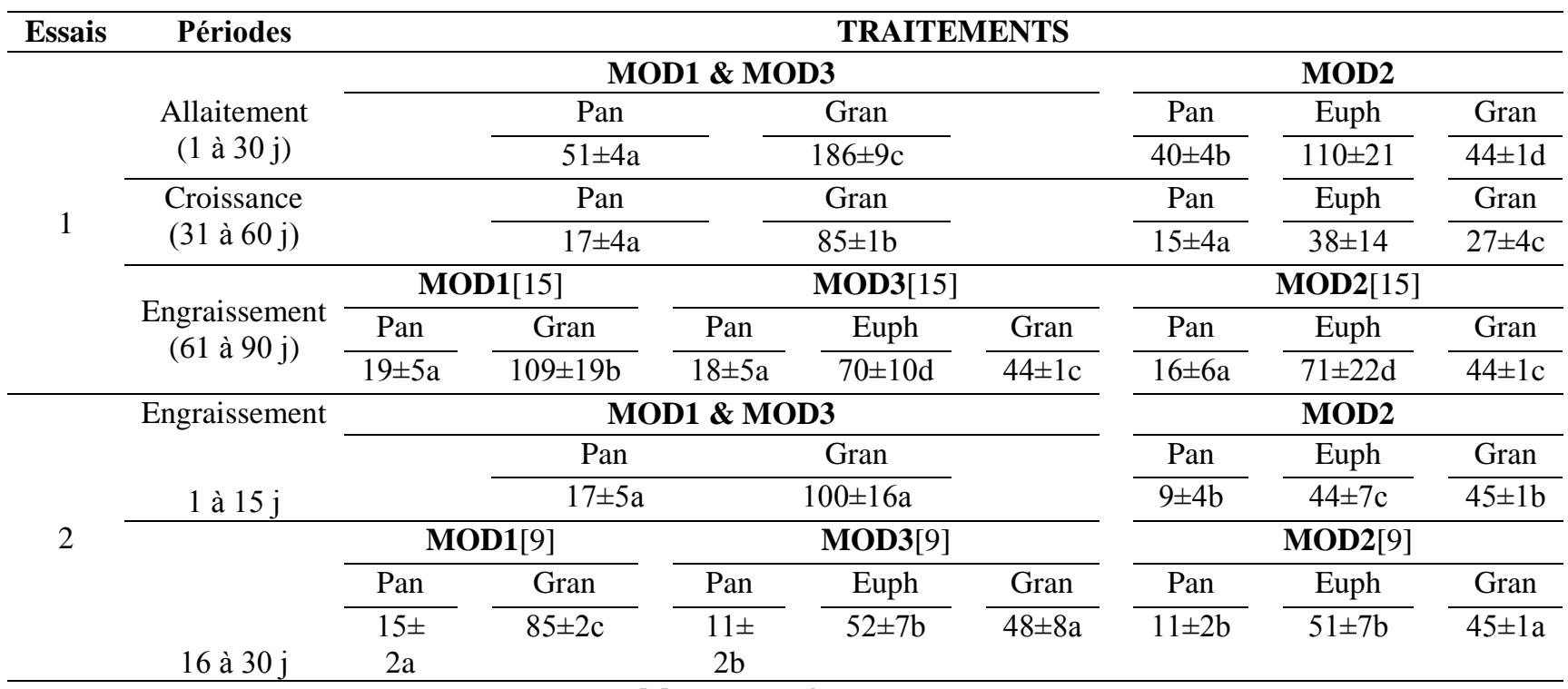

Moyennes \pm écart-type

a, b, c, les moyennes de la même ligne suivies de la même lettre du même aliment ne sont pas significativement différentes.

MOD1 : Distribution du P. maximum associé au granulé pour lapin ad libitum durant 30 jours ;

MOD2: Distribution du P. maximum associé à E. heterophylla ad libitum avec une quantité de granulé pour lapin de $50 \mathrm{~g}$ durant 30 jours ;

MOD3: Pangran pendant 15 jours suivi de Paneuh les 15 derniers jours).

Pan : P. maximum; Euph: E. heterophylla; Gran: Granulé pour lapin; [ ] = Nombre d'animaux

\section{Effet du régime sur la cholestérolémie}

Dans l'Essai 1, les taux de cholestérol total des lapins différaient significativement selon les régimes, tout comme ceux du cholestérol HDL $(\mathrm{P}<0,05)$. L'ingestion d'E. heterophylla a induit une baisse du cholestérol total de 40,3 et 56,3\% et une réduction du cholestérol HDL de 14,7 et 39,6\% respectivement après 30 jours et 90 jours. Les teneurs de triglycérides des régimes supplémentés en $E$. heterophylla représentaient environ la moitié de celle du régime non supplémenté $(\mathrm{P}<0,05)$. Quant aux taux de cholestérol LDL des animaux soumis aux différents régimes, ils différaient significativement les uns des autres $(\mathrm{P}<0,05)$. Dans cet essai, une réduction significative du cholestérol LDL de $26 \%$ et $60 \%$ respectivement après 30 jours et 90 jours d'ingestions d'E. heterophylla a été observée (Tableau 5). 
Dans le second essai, les taux de cholestérol total et de cholestérol LDL des lapins soumis aux traitements contenant E. heterophylla étaient significativement inférieurs à ceux des lapins soumis au traitement témoin (MOD1) $(\mathrm{P}<0,05)$. L'ingestion d'E. heterophylla a induit une baisse du cholestérol total de 30 et $45,1 \%$ et une réduction du cholestérol LDL de $59,2 \%$ et de $65,4 \%$ respectivement après 15 jours et 30 jours. Les taux de cholestérol HDL des animaux soumis au traitement MOD2 étaient 31 et $43 \%$ plus faibles que ceux des traitements MOD3 et MOD1 respectivement $(\mathrm{P}<0,05)$, qui eux ne différaient pas significativement entre eux. Les teneurs de triglycérides ne différaient pas selon les traitements $(\mathrm{P}>0,05)$. Par ailleurs dans cet essai, il ressort que deux semaines d'ingestion d'E. heterophylla suffisent à réduire significativement les teneurs en cholestérol total et en cholestérol LDL chez les lapins locaux (Tableau 5).

\section{Discussions}

\section{Choix méthodologique}

La détermination de la teneur en cholestérol plasmatique chez les animaux après ingestion d'un régime contenant des plantes à forte potentialité hypocholestérolémiante est une pratique courante en production animale (Nuhu, 2010 ; Rohilla et al., 2011). Cette méthode permet de montrer que l'incorporation de ressources non conventionnelles dans l'alimentation des animaux, induit une réduction du cholestérol plasmatique et par la suite, celle du cholestérol des produits animaux (muscle et jaune d'œuf). Elle diffère de celle pratiquée en pharmacologie qui consiste en l'obtention de différentes concentrations d'extraits de substances naturelles dans des solutions aqueuses, de l'alcool, etc.., puis d'en déterminer les effets sur le cholestérol plasmatique chez les animaux (Gohil et al., 2013).

Tableau 5 : Concentrations en cholestérol et triglycérides chez les lapins locaux en fonction des traitements étud

\begin{tabular}{lllll}
\hline \multirow{2}{*}{ Essais } & \multirow{2}{*}{ Paramètres sériques $(\mathrm{mg} / \mathrm{dl})$} & \multicolumn{3}{l}{ Traitements étudiés } \\
\cline { 3 - 5 } & & MOD1 & MOD2 & MOD3 \\
\hline \multirow{4}{*}{1} & Cholestérol total & $56,8 \pm 6,8 \mathrm{a}$ & $24,8 \pm 5,5 \mathrm{~b}$ & $33,9 \pm 7,9 \mathrm{~b}$ \\
& Cholestérol-HDL & $24,5 \pm 5,4 \mathrm{a}$ & $14,8 \pm 5,1 \mathrm{a}$ & $20,9 \pm 7,0 \mathrm{a}$ \\
& Triglycérides & $73,2 \pm 16,4 \mathrm{a}$ & $34,2 \pm 2,2 \mathrm{~b}$ & $30,9 \pm 7,6 \mathrm{~b}$ \\
& Cholestérol-LDL & $17,7 \pm 1,8 \mathrm{a}$ & $3,2 \pm 1,7 \mathrm{~b}$ & $6,8 \pm 1,4 \mathrm{c}$ \\
\hline \multirow{4}{*}{2} & Cholestérol total & $41,2 \pm 6,5 \mathrm{a}$ & $22,6 \pm 2.7 \mathrm{~b}$ & $28,8 \pm 6,0 \mathrm{~b}$ \\
& Cholestérol-HDL & $26,7 \pm 4,0 \mathrm{a}$ & $15,0 \pm 4,1 \mathrm{~b}$ & $21,8 \pm 2,0 \mathrm{a}$ \\
& Triglycérides & $24,1 \pm 1,3 \mathrm{a}$ & $27,9 \pm 3,6 \mathrm{~b}$ & $19,8 \pm 1,2 \mathrm{c}$ \\
& Cholestérol-LDL & $13,0 \pm 1,2 \mathrm{a}$ & $4,5 \pm 2,1 \mathrm{~b}$ & $5,3 \pm 1,5 \mathrm{~b}$ \\
\hline
\end{tabular}

Moyenne \pm écart-type de 6 lapins

a, b, c, les moyennes de la même ligne suivies de la même lettre ne sont pas significativement différentes. 


\section{Propriétés hypocholestérolémiantes d'E. heterophylla}

Au regard des résultats obtenus dans le sérum des lapins, il semble indéniable que E. heterophylla possède des propriétés hypocholestérolémiantes. A notre connaissance, c'est la première étude qui montre cet effet chez l'animal. Des résultats similaires ont été obtenus avec E. hirta, une plante médicinale indigène (Huang et al., 2012). Une des raisons susceptibles d'expliquer le manque d'information sur les potentialités hypocholestérolémiantes d'E. heterophylla, pourrait être la toxicité signalée de cette plante chez l'homme par voie orale (Falodum et al., 2003). Une toxicité qu'il faudrait relativiser car elle pourrait dépendre de la dose, du mode de traitement, de l'organe de la plante, du modèle animal et de l'état physiologique de l'individu (Nalule et al., 2017 ; Prota, 2016).

\section{Substances hypocholestérolémiantes d' $E$. heterophylla}

Les teneurs en acide gras alpha-linolénique (C18:3 n-3), auraient contribué à la modification du profil lipidique du sérum des lapins, permettant ainsi une réduction du risque d'athérosclérose et de maladies cardiovasculaires. Cependant, au regard des pourcentages de réductions de la cholestérolémie, il semble exister inéluctablement une synergie de plusieurs mécanismes d'action de différents métabolites primaires (Protéines, hydrates de carbone), de métabolites secondaires, notamment le stigmastérol et le $\beta$ stigmastérol glucoside (Falodum et al., 2008), des polyphénols tels que les saponines, les flavonoïdes, les alcaloïdes, les phénols, les tannins et l'acide ascorbique (James et Friday, 2010 ; Faleye et al., 2012 ; Keerhana, 2014). Trois mécanismes d'action probables de ces molécules pourraient être mis en œuvre : (i) l'inhibition de l'absorption et de la réabsorption intestinale du cholestérol avec une augmentation de l'élimination fécale des acides biliaires (Dubuc, 2010), (ii) la protection contre la péroxydation avec l'action des antioxydants et (iii) l'inhibition de la synthèse du cholestérol (Raisonnier, 2004).

\section{Effets hypocholestérolémiantes $E$. heterophylla}

La teneur moyenne du cholestérol total enregistrée lors de l'Essai 1 est conforme à celles de plusieurs auteurs $(0,55 \mathrm{~g} / \mathrm{l})$ obtenues dans le sérum des lapins (Kheffach, 2015 ; Adéoti et al., 2013). Par contre, elle est plus élevée que celles obtenues $(47,87 \mathrm{mg} / \mathrm{dl})$ par Othmani-Mecif et Benazzoug (2005) chez des lapines non gestantes. Ces résultats confirment ceux obtenus au niveau de l'Essai 2. Les réductions de $45 \%$ du cholestérol total sérique corroborent les résultats obtenus par Ogbuewu et al. (2008) (50,77\%), après l'introduction de $15 \%$ des feuilles de margousier dans l'alimentation des lapins. Par ailleurs, comparée aux feuilles de Leucaena leucocephala, E. heterophylla semble être une plante nettement plus hypocholestérolémiante. 
En effet, pour analyser les effets hématologiques et biochimiques de $L$. leucocephala, Rohilla et al. (2011) ont incorporé $20 \%, 40 \%$ et $60 \%$ des feuilles de cette plante dans l'alimentation des lapins. Les résultats obtenus après trois mois de traitement ont montré que le meilleur taux d'incorporation des feuilles de cette plante dans l'alimentation des lapins était de $40 \%$ avec une réduction du cholestérol total et du cholestérol LDL respectivement de $8,7 \%$ et $3,7 \%$.

\section{Conclusion}

Ce travail avait pour objectif d'évaluer le pouvoir hypocholestérolémiant d'E. heterophylla. Les deux essais réalisés ont montré une réduction significative du cholestérol LDL ou "mauvais cholestérol". Ces résultats obtenus chez des lapins locaux montrent que E. heterophylla contiendrait des molécules hypocholestérolémiantes qu'il faudrait mieux identifier et quantifier.

\section{References:}

1. Abegunde, D. O., Mathers, C. D., Adam, T., Ortegon, M. \& Strong, K. (2007). The burden and costs of chronic diseases in low-income and middle-income countries. Lancet, (-370), 1929-1938.

2. Adeoti, M., Djyh, B., Djaman, A. \& Sess, E. (2013). Etude des variations du pool des lipides et des marqueurs de la lipopéroxydation chez le lapin sous traitement par l'extrait chloroformique de Mansonia altissima. Revue Bio-Africa, (11), 7-12.

3. Ahongo, Y. D., Kouakou, N. D.V., Angbo, C. E. M., Assidjo, E. N. \& Kouba, M. (2016). Effect of a diet supplemented with Euphorbia heterophylla on lipid parameters in the thighs of rabbits. In: European Annual Animal Production Book, Wageningen, Pays Bas, p562.

4. AOAC (2006). Presidential task force on best practices for microbiological methodology. BPMM Task Force Final Report 8-706, p201.

5. Barcelo-Coblijn, G. \& Murphy, E. J. (2009). Alpha linolenic and its conversion to longer chain n-3 fatty acids: Benefits for human health and a role in maintaining tissue n-3 fatty acid lavels. Prog. lip. rech, (48), 355-374.

6. Callias, C. (2007). Les alicaments dans la lutte contre l'hypercholestérolémie. Bulletin de la Société des Enseignants Neuchâtelois de Sciences, (30), p18.

7. Dubuc, G. (2010). La PCSK9 humaine: une molécule aux multiples facettes métaboliques et une cible thérapeutique prometteuse études de régulation in vitro et in vivo. Thèse de Philosophiae Doctor (Ph.D.) en sciences biomédicales. Université de Montréal, p233. 
8. Dylan, S. M. \& Peter, J. J. (2010). Le rôle des phytostérols et les maladies cardiovasculaires. Université du Manitoba, Canada. Département des Sciences de l'alimentation. La Revue WhitehallRobins, 19 (1), p2.

9. Faleye, F. J., Odeyemi, A. T. \& Aderogba, A. A. (2012). Evaluation of the chemical composition and antimicrobial activities of three Nigerian medicinal plants. Elixir Appl. Biology, (45), 7652-7656.

10. Falodun, A., Agbakwuru, E. O. P. \& Ukoh, G. C. (2003). Antibacterial activity of Euphorbia heterophylla linn (family Euphorbiaceae) Pak. J. Sci. Res., 46 (6), 471-472.

11. Falodun, A., Ali S., Quardir, M. I. \& Choudhary, M. I. I. (2008). Phytochemical and biological investigation of chloroform and ethylacetate fractions of Euphorbia heterophylla leaf (Euphorbiaceae). J. Med. Plant. Res., 2 (12), 365-369.

12. FAO (2015). Maladies cardiovasculaires .Aide-mémoire Janvier 2015. Consulté le 15/09/15

13. Friedewald, W. T., Levy, R. I. \& Fredrickson, D. S. (1972). Estimation of the concentration of low-density lipoprotein cholesterol in plasma, without use of the preparative ultracentrifuge. Clin Chem., (18), 499-502.

14. Gohil, V. P., Shah, B. G. \& Sukhval, S. H. (2013). Effect of stigmasterol against high-fat diet-induced dyslipidemia in rats: A preliminary study. Scholar Reseach Library. Der Pharmacia Lettre, 5 (4), 305-309.

15. Huang, L., Chen, S. \& Yang, M. (2012). Euphorbia hirta (Feiyangcao): A review on its ethnopharmacology, phytochemistry and pharmacology. J. Med. Plants Res., 6 (39), 5176-5185.

16. Iserin, P., Moulard, F., Rachel, R., Biaujeaud, M., Ringuet, J., Bloch, J., Ybert E., Vican, P., Masson, M., Moulard, F., Restellini, J-P. \& Botrel, A. (2001). La rousse : encyclopédie des plantes médicinales ; identification, préparation, soins. 2 éd, Paris, 155-291.

17. James, O. \& Friday, E. T. (2010). Phytochemical composition, bioactivity and wound healing potential of Euphorbia heterophylla (Euphorbiaceae) leaf extract. Inter. J. Pharm. Biom. Res., 1 (1), 5463.

18. Keerthana, K., Deepa, A., Shobana, G., Jothi, G. \& Sridharan, G. (2014). Preleminary phytochemical screening and in vitro antioxidant potential of Euphorbia heterophylla. Int J Pharm Pharm Sci., 6 (8), 549-553.

19. Kenfack, A., Tchoumboué, J., Kamtchouing, P. \& Ngoula, F. (2006). Effets de la substitution par l'arachide fourragère (Arachis glabrata) de l'herbe à éléphant (Pennisetum purpureum) sur le nombre 
d'ovulations et les mortalités prénatales chez le cobaye (Cavia porcellus L.) adulte. Tropicultura, 24 (3), 143-146.

20. Kheffach, A. (2015). La cytotoxicité de certaines huiles essentielles chez les lapins. Mémoire de de Master. Université Echahid Hamma Lakhdar D'el-Oued, p161.

21. Kouakou, N. D. V., Cheick, T. G., Angbo-Kouakou, C. E. M., Kouamé, K. B., Adima, A. A., Assidjo, N. E., Grongnet, J-F. \& Kouba, M. (2015). Essai préliminaire de production d'œufs des poules pondeuses (ISA Warren) enrichis en acides gras polyinsaturés oméga 3 avec les graines de Euphorbia heterophylla L. International Formulae Group. Int. J. Biol. Chem. Sci, 9 (4), 1902-1909.

22. Kouakou, N. D. V., Coulibaly, S. B. M., Angbo-kouakou, C. E. M., Thys, E., Assidjo, N. E. \& Kouba, M. (2016). Réduction des coûts alimentaires des lapins (Oryctolagus cuniculus L.) par la distribution de l'herbe de lait (Euphorbia heterophylla (L.) Klotz. \& Garcke) associée à l'herbe de Guinée (Panicum maximum Jacq.) Lam. en élevage semi-intensif. J. App. Bios., (99), 9373-9381.

23. Kouakou, N. D.V., Grongnet, J-F., Assidjo, N. E., Thys, E., Marnet, P-G., Catheline, D., Legrand, P. \& Kouba, M. (2013). Effect of a supplementation of Euphorbia heterophylla on nutritional meat quality of Guinea pig (Cavia porcellus L.). Meat Science, 93 (4), 821-826.

24. N'Guessan, K., Soro, D. \& Amon, A. D. E. (2011). Plantes utilisées en médecine traditionnelle dans le traitement des maladies cardiovasculaires, en pays Abbey et Krobou, dans le Sud de la Côted'Ivoire. Ecthnopharmacologie, Phytothérapie, 9 (4), 199-208.

25. Nalule, A. S., Afayoa, M., Malim, B. \& Majidu, M. (2017). Acute oral toxicity of Euphorbia heterophylla Linn. ethanolic extract in albino mice. Afri. J. Pharm. Pharm., 11(1), 1-9.

26. Nuhu, F. (2010). Effect of moringa leaf meal (MOLM) on nutrient digestibility, growth, carcass and blood indices of weaner rabbits. In: MSc. Thesis. Anim. Nutr., Kwame Nkrumah University, Kumasi (Ghana), p122.

27. Ogbuewu, I. P., Okoli, I. C. \& Iloeje, M. (2008). Serum biochemical evaluation and organ weight characteristics of buck rabbits fed graded levels Neem (Azadirachta indica) leaf meal diets. Veton - line - The International Journal of Veterinary Medicine 2008.

28. OMS (2003). Organisation mondiale de la Santé. Rapport sur la santé dans le monde. 1211 Genève 27, Suisse, www.who.int/whr/2003/en/whr03_fr.pdf. Consulté le 16/10/2016.

29. Othmani-Mecif, K. \& Benazzoug, Y. (2005). Caractérisation de certains paramètres biochimiques plasmatiques et histologiques 
(tractus génital femelle) chez la population locale de lapin (Oryctolagus cuniculus) non gestante et au cours de la gestation. Sciences et technologie, (23), 91-96.

30. Prota (2016). Euphorbia heterophylla (PROTA). (2014, décembre 17). Plant Use. Retrieved 22:38, novembre 13, 2016 depuis http://uses.plantnet

project.org/f/index.php?title=Euphorbia_heterophylla_(PROTA)\&old $\mathrm{id}=61043$.

31. Raisonnier, A. (2004). Lipides et lipoprotéines. Cours de Biochimie Métabolique et Régulations. Université Pierre et Marie Curie, p106

32. Rohilla, P.P., Bujarbaruah, K. M., Kumar, M. \& Singh (2011). Haematological and biochemical responses of various levels of Subabul (Leucaena leucocephala) leaves in growing rabbits. Indian J. Anim. Nutr., 17 (1), 28-33.

33. Scheen, A. J. (2011). Les statines dans la prise en charge des dyslipidémies. Université de Liège, département de médecine, Revue du Praticien, (6), 1120-1126. 